\title{
An Extension of the Dang Van Fatigue Criterion to Finite Life Predictions
}

\author{
A.M. Korsunsky, G. Peridas, D.A. Hills \\ Department of Engineering Science. University of Oxford \\ Parks Road, Oxford OX1 3PJ, England \\ Corresponding author: alexander.korsunsky@eng.ox.ac.uk
}

\begin{abstract}
An extension to Dang Van's crack initiation and Sines' failure criterion is formulated permitting finite life predictions to be made. This draws on the recent results regarding the relationship between the Dang Van criterion, Sines' criterion and Goodman's rule. The approach is applied to a set of experimental data obtained by Socie /1/. The results show a good correlation between predictions and experiment, suggesting that the Dang Van criterion modified in this way can usefully scrve to predict HCF life. In order to establish which of the two criteria performs best, data spanning a wider range of $\mathrm{R}$-ratios is required.
\end{abstract}

\section{INTRODUCTION}

Studies aimed at establishing a correlation between bulk multiaxial, non-proportional stress states and crack initiation have been reported, examples being the criteria due to Crossland and Sines et al $/ 2 /$. To incorporate significant stress non-uniformity and plasticity effects, most modern approaches to the prediction of fatigue crack initiation were developed on the basis of the 'critical plane' concept: crack initiation is most likely at the location and in the orientation for which the most severe conditions are found. Often the conditions are expressed in terms of combinations of stresses and strains, some of the best known being due to Smith, Watson and Topper $/ 3 /$, and Fatemi and Socie /4/. A distinct disadvantage of the critical plane approaches for problems involving complex geometries and loading paths is that they require full field elasto-plastic solution on a cycle-by-cycle basis, as the plastic strain as well as stress is required in the calculation. This often renders them inefficient in the context of an iterative design procedure of a complex component. 
Dang Van et al $/ 5,6 /$ made an attempt to put the analysis of the crack initiation process on a fundalnental basis while retaining the traditional 'bulk' correlation approach. The advantage over the critical plane approaches is that it allows the use of suitable calibration experiments to take into account surface finish effects, or differences in grain structures, or, possibly, the different mechanics of initiation active under special conditions (for example, contact fatigue). Dang Van's crack initiation criterion is based on clear physical reasoning about micro-scale stress localisation, and in its original form Dang Van's criterion provides a 'go/no go' condition for nucleation. The criterion states that no crack will initiate if the material undergoes complete elastic shakedown at the grain level. Hence, the boundary between infinite and finite life can be drawn. No elasto-plastic solutions are required for this: an elastic solution together with a simple, computationally undemanding algorithm is sufficient for the determination of the critical quantities.

Within the present study the Dang Van concept was extended to determine finite nucleation lives in problems where nucleation occurs. Firstly, the accuracy of the criterion was optimised by devising the means of obtaining a wider spread of points from calibration experiments. Secondly, the relationship was investigated with traditional bulk fatigue approaches, notably Sines' criterion and Goodman's rule. Thirdly, the criterion was modified and extended so as to obtain finite life predictions (for initiation dominated situations where propagation times are insignificant). Finally, the theory was tested and validated against experimental data.

The basis of the Dang Van criterion is the following: 'No crack is expected to initiate if shakedown to an elastic state occurs at the grain level'. If, therefore, all grains in the material are in the elastic state after the first few loading cycles, we expect an infinite life. Unfortunately, it is very difficult or impracticable to measure or predict grain-level stresses, and so a pseudo-shaken-down grain-level stress state based on the bulk stress state is estimated instead and compared to data obtained from calibration experiments. Thus, Dang Van's criterion is expressed as essentially a bulk corrclation based on the properties of the stress state (maximum shear stress and hydrostatic pressure, Figure 1). Originally Dang Van addresses solely the question of crack initiation. However, for Iligh Cycle Fatigue applications where propagation times are negligible compared to initiation times, the criterion can be extended to predict the time to failure,

In Dang Van's criterion a linear relationship between maximum shear stress and hydrostatic pressure is assumed to delineate the fatigue limit. By considering the effects of R-ratio on Dang Van's parameter it can be noted that, under uniaxial conditions, Dang Van's criterion and Goodman's rule should give identical predictions $/ 7 /$. Of course, Goodman's rule is applicable to finite life predictions, and has been used in this context for some time. The correlation established between the two criteria is the basis for extending Dang Van's criterion to finite life predictions.

In the present study we compare the predictions with experimental results and examine the nature of the correlation. This enables Dang Van's criterion to be extended to finite life predictions for a general multiaxial stress state. This could prove very useful in rapid life predictions of both simple and complex components, where an analytically or numerically obtained elasticity solution can be mapped onto the Dang Van diagram 
in order to obtain an estimate for the total life in a computationally inexpensive way. To assess the value of this approach, it was also compared to the predictions of a traditional bulk fatigue criterion by Sines.

\section{EXPERIMENTAL DATA}

A set of data from the experiments carried out by Socic /1/ was used. The tests were performed on AISI Type 304 stainless steel tubular specimens subjected to tension and torsion. The specimens were subjected to six different cyclic load histories, denoted A, B, C, N, P and Q. Within each class of loading histories the stress magnitudes were scaled proportionally to each nominal loading case. Experiment specifications and useful material properties are given in Tables 1 and $2 / 1 /$. The loading cases produce various paths in $\tau_{\max }-\bar{\sigma}$ space that are sufficiently different from each other to examine the Dang Van - based initiation hypothesis. Waveforms were nominally symmetrical, but small offsets in tension or torsion may have been present during the experiments.

Table 1

Experiment Specifications

\begin{tabular}{|c|c|c|c|c|c|c||}
\hline $\begin{array}{c}\text { WAVEFORM/ } \\
\text { SPECIMEN }\end{array}$ & $\begin{array}{c}\text { NUMBER OF } \\
\text { SPECIMENS }\end{array}$ & $\begin{array}{c}\text { MEAN } \\
\text { NORMAL } \\
\text { STRESS }\end{array}$ & $\begin{array}{c}\text { NORMAL } \\
\text { STRESS } \\
\text { RANGE }\end{array}$ & $\begin{array}{c}\text { MEAN } \\
\text { SHEAR } \\
\text { STRESS }\end{array}$ & $\begin{array}{c}\text { SHEAR } \\
\text { STRESS } \\
\text { RANGE }\end{array}$ & $N_{f}$ \\
\hline A2 & 10 & 0 & 558 & 0 & 0 & 10300 \\
\hline B4 & 9 & 0 & 0 & 0 & 314 & 33900 \\
\hline C3 & 4 & 0.6 & 322 & 1.2 & 178 & 444000 \\
\hline N1 & 4 & 2.6 & 954 & -4.4 & 534 & 3560 \\
\hline P1 & 4 & 0 & 840 & 0 & 452 & 5110 \\
\hline Q1 & 4 & 32 & 730 & -13.2 & 398 & 11080 \\
\hline
\end{tabular}


Table 2

Material Properties

\begin{tabular}{|c|c|c|}
\hline MATERIAI. PROPERTIES & & \\
\hline Young's Modulus & $183 \mathrm{MPa}$ & \multirow{3}{*}{$\begin{array}{c}\text { MONOTONIC TENSILE } \\
\text { PROPERTIES }\end{array}$} \\
\hline $0.2 \%$ Offset Yield Stress & $325 \mathrm{MPa}$ & \\
\hline Ultimate Tensile Strength & $650 \mathrm{MPa}$ & \\
\hline Fatigue Strength Coeflicient (C) & $1000 \mathrm{MPa}$ & \multirow{2}{*}{$\begin{array}{l}\text { AXIAL TENSILE } \\
\text { PROPERTIES }\end{array}$} \\
\hline Fatigue Strength Coefficient (n) & 0.114 & \\
\hline Fatigue Strength Coefficient (C) & $709 \mathrm{MPa}$ & \multirow{2}{*}{$\begin{array}{c}\text { TORSIONAL CYCLIC } \\
\text { PROPERTIES }\end{array}$} \\
\hline Fatigue Strength Coefficient (n) & 0.121 & \\
\hline
\end{tabular}

\section{METHOD OF ANALYSIS}

A set of data from the experiments carried out by Socie /1/ was used. The tests were performed Dang Van's method seeks a local value of residual bulk stress (not a spatially equilibrating ficld) which, when superimposed on the applied stress state, will minimise the tendency to yield. $A$ pseudo-shakedown calculation is carried out to find a new origin in 6-dimensional deviatoric stress space $\left(s_{11} / \sqrt{2}, s_{22} / \sqrt{2}, s_{33} / \sqrt{2}, s_{12}, s_{13}, s_{23}\right)$ with respect to which the maximum value of $J_{2}$ obtained around the path is minimised. This stress state with respect to this new origin is termed the microscopic (grain level) stress state. The maximum shear stress $\tau_{\max }$ and the hydrostatic pressure $\bar{\sigma}$ are calculated, and plotted on the $\tau_{\max }-\bar{\sigma}$ diagram. A comparison is then made with the fatigue limit line, which is assumed to be given by:

$\tau_{\max }+a \bar{\sigma}=b$

Parameters $a$ and $b$ are calibrated using simple experiments like pure torsion and pure bending. 


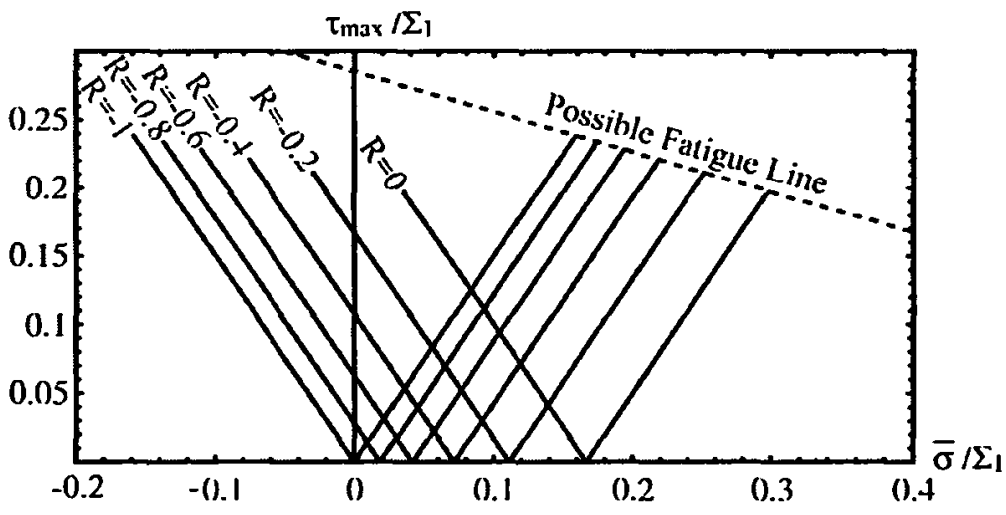

Fig. 1: Mean stress and range effects on the Dang Van diagram

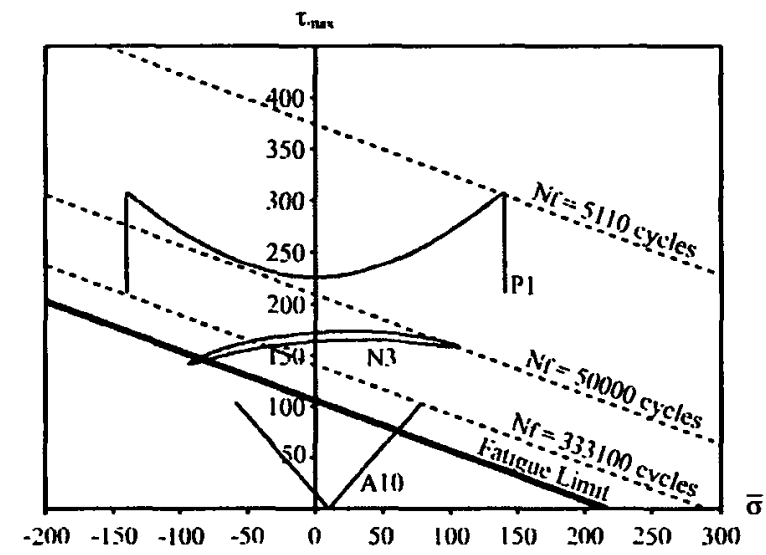

Fig. 2: Illustration of the load paths in the Dang Van plane for

some waveforms

A simple analysis reveals that, under uniaxial conditions, the Dang Van criterion predicts behaviour identical to that in Goodman's rule: the allowable alternating stress decreases linearly as the mean static stress increases. This may be visualised if we note that the locus produced in $\tau_{\max }-\bar{\sigma}$ space by an oscillating uniaxial load is a V-shape (Figure 1). The size of the $V$ is directly proportional to the amplitude of the load, while its position along the horizontal axis is determined by the degree of load reversal ( $R$-ratio). Therefore, to ensure that all points on the $V$ remain below the fatigue line, the amplitude must decrease with increasing $R$-ratio. The resulting relationship between alternating (range) and mean stress is linear, as in the Goodman's rule /7/: 
$\sigma_{a}=\sigma_{0}\left(1-\frac{\sigma_{m}}{\sigma_{*}}\right)$

where $\sigma_{*}$ can be set to a value between the jield stress, $\sigma_{r}$, and the ultimate tensile strength, $\sigma_{1 r \text { s }}$. The relationship between the criteria can be found as:

$$
a=\frac{3 \sigma_{0}}{2\left(\sigma_{*}-\sigma_{0}\right)}, \quad b=\frac{\sigma_{*} \sigma_{0}}{2\left(\sigma_{*}-\sigma_{0}\right)}
$$

This correlation provides a means of extending Dang Van's Criterion to finite life predictions. Although Goodman's Rulc was originally formulated for the infinite-finite life boundary, it has since been used successfully for finite lives. A family of lines representing the locus of failure at different numbers of cycles can be constructed, e.g. as a fan following Goodman's recipe, or parallel to the boundary between infinite and finite lives. The original Dang Van criterion is concerned with crack initiation and not the total life. We make the assumption that under ICF the majority of life is spent initiating a crack, so that once that happens subsequent propagation time to failure is insignificant. We therefore opt for the parallel lines approach, noting that for HCF situations the critical point on the Dang Van diagram usually lies at high values of $\tau_{\max }$ and low values of the hydrostatic pressure $\bar{\sigma}$. where the difference between a fan and a parallel family of lines is not very significant.

While Goodman's rule provides a method of estimating finite life in a uniaxial context, the reasoning underlying the Dang Van approach is multiaxial. A similar connection has been established between Dang Van's criterion and Sines' bulk fatigue criterion /7/. Here we correlate Goodman's rule and Sines' criterion, the motive being to extend the Dang Van principle to predict finite initiation lives. Fatigue limit in Sines space is given by:

$$
\sqrt{J_{2_{\text {att }}}}+A I_{1_{\text {sut }}}=B
$$

where $J_{2_{\text {ult }}}$ is the maximum value of the second deviatoric stress invariant over the load path, and $I_{1_{\text {utut }}}$ is the first stress invariant of the static component of stress. Using the simple relationship between the Goodman variables $\left(\sigma_{a}\right.$ and $\left.\sigma_{m}\right)$ and Sines variables $\left(J_{2_{\text {utt }}}\right.$ and $\left.I_{1_{\text {sutum }}}\right)$, Sines' fatigue limit's constants are found as:

$$
A=\frac{\sigma_{0}}{\sqrt{3} \sigma_{*}}, \quad B=\frac{\sigma_{0}}{\sqrt{3}} .
$$


The shakedown calculation to find the optimal residual stress state for each of the cycles was carried out with the help of Mathematica, employing the algorithm described in $/ 5 /$. First, the bulk hydrostatic pressure and deviatoric stresses are worked out, and then the optimal residual stresses are sought such that the load path in deviatoric stress space is encompassed by the smallest possible hypersphere. The centre of the hypersphere corresponds to the residual stresses sought. The algorithm may start by assuming a small radius and an arbitrary centre for the hypersphere (e.g. the centroid of the loading path) and then correcting the values for centroid and radius as the load path is traversed sequentially from point to point, until the algorithm converges to the desired residual stress. The speed of convergence can be improved by directly seeking the largest diameter in the load path.

In some cases residual stresses may be found by inspection. This is commonly the case if the loading path in the deviatoric stress space is straight. The load path in this case is a diameter of the hypersphere, and its midpoint the centre. Examples of such cases are waveforms A and B in Socie's data set, while N, P, Q corresponded to more complex paths (Figure 2). For load histories that exhibit symmetry about the origin in the $\sigma-\tau$ space, the deviatoric load path is symmetric about the origin and hence the residual stresses are zero. However, the use of the algorithm was necessary for some of the Socie data examined which showed some small shift from the symmetric conditions of zero static stress $(R=-1)$. Finally, comparison with Sines'

criterion is straightforward, since the stress history allows one to calculate $I_{1_{\text {uat }}}$ and the maximum value of $\sqrt{J_{2} \text { att }}$ over the cycle.

\section{ANALYSIS}

Based on the identification of the critical points on the $\tau_{\max }-\bar{\sigma}$ diagram for the data, the finite life postulate based on the Dang Van criterion can be assessed in comparison with Sines' criterion. Socie gives the Basquin's Law constants for the material in simple tension and simple torsion which are here reproduced in Table 2 in the form

$\Delta \sigma N_{f}^{n}=C$

Setting $N_{f}$ to be $10^{7}$ (as is customary for the boundary between finite and infinite life for such materials) and using values for $n$ and $C$ from Table 2 above, an estimate for the fatigue limit in simple tension is obtained as $159 \mathrm{MPa}$. Using the relationship between Goodman constants, and Dang Van and Sines constants of Eq. (3) and (5) one can calculate the slope of the fatigue limit line, $a$, in the Dang Van space, and $A$, in Sines' space. Lines corresponding to finite lives are now constructed with the same slope, through all the data 
points calculated before. Thus, life corresponding to each line is known. A smooth decrease in fatigue life is expected. from the high values near the fatigue limit and towards lower values the further away we move in a perpendicular direction. To assess the performance of this finite life Dang Van criterion, a plot is constructed of perpendicular distance from the origin. $d$ (Dang Van space) or $D$ (Sines space), vs. number of cycles to failure on a logarithmic scale, $N$. The quantities $d$ and $D$ are easily calculated as:

$$
d=\frac{-b+\tau_{\max }+a \bar{\sigma}}{\sqrt{1+a^{2}}} . \quad D=\frac{-B+\sqrt{J_{2_{a d u}}}+A I_{1_{\mathrm{sum}}}}{\sqrt{1+A^{2}}}
$$

The Dang Van analysis for waveforms A and B above can be used to map Basquin's law onto Dang Van space $\left(\tau_{\max }-\bar{\sigma}\right)$. Then, using the value of $a$, the result is mapped onto a $d-N_{f}$ diagram.

Figure 3 shows all the data sets in $d-N_{f}$ space. It can be seen that the Basquin lines for tension and torsion are almost coincident, indicating that the procedure has a firm footing. The agreement between Basquin and the experimental data is also excellent. A power-law function was fitted to the data points, and this fit can be seen to resemble closely the Basquin lines. The scatter exhibited is acceptable for fatigue data of this nature. Good agreement can be also observed between data points of the same waveform, although only waveforms $A$ and $B$ have enough data points for a meaningful comparison.

Basquin's Law may be also mapped onto Sines space. Since the Law represents fully reversing loading.

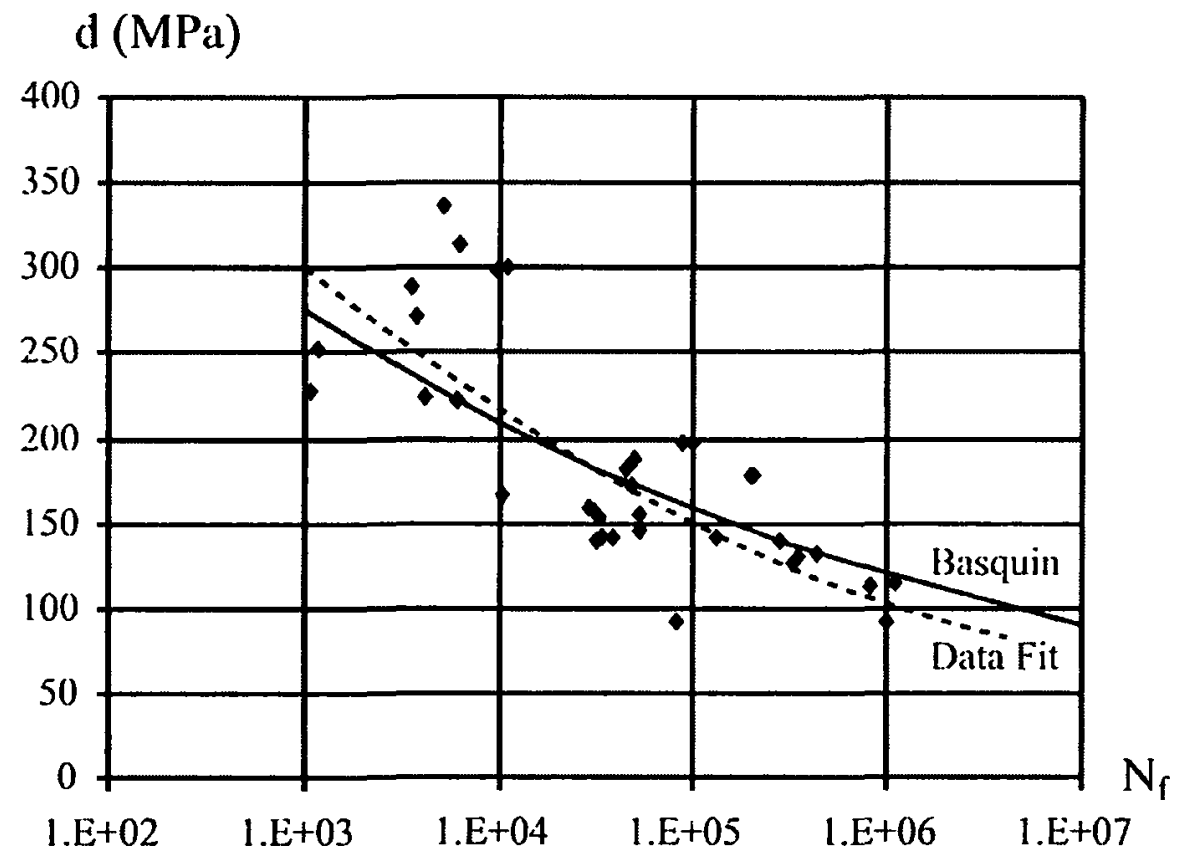

Fig. 3: The Dang Van correlation for finite fatigue lives for a large collection of non-proportional complex loading paths. 
$I_{1_{\text {sat }}}$ is zero, whereas $\sqrt{J_{2_{\text {alt }}}}$ is calculated from the stress values. Using the value of $A$, this is then mapped onto a $D-N_{f}$ diagram. Basquin's law for tension and torsion do not show as close an agreement between them according to Sines' criterion, as they do in the Dang Van space.

The fact that the static components of stress are very small implies that the pseudo-shakedown procedure taking place according to Dang Van analysis is minimal. Ideally, a set of data exhibiting a higher degree of asymmetry about the origin would be required to test fully the extended finite life Dang Van procedure and also highlight any difference between it and traditional criteria like Sines'. The authors expect that such a comparison would favour an approach like Dang Van due to the pseudo-shakedown procedure. The conclusion from the above analysis is that for small static stresses, the described method of extending Dang Van's and Sines' criteria to finite life predictions yields a good correlation in both cases, although slightly better in the former.

\section{CONCLUSIONS}

The analytical framework for extending Dang Van's and Sines' criteria to finite life predictions has been presented based on a relationship between Dang Van, Sines and Goodman constants. A set of experimental data by Socie $/ 1 /$ was analysed. For the six types of experiments, Dang Van $\left(\tau_{\max }, \bar{\sigma}\right)$ and Sines $\left(J_{2_{\text {att }}}, I_{1_{\text {rat }}}\right)$ quantities were calculated. A line with a slope found from the Goodman diagram was drawn through each data point in $\tau_{\max }-\bar{\sigma}$ and $J_{2_{u l t}}-I_{I_{\text {sut }}}$ space, and subsequently the perpendicular distance of each line from the origin defined by Eq. (7) plotted against number of cycles to failure. The same procedure was carried out for Basquin's Law for pure tension and pure torsion using constants given in $/ 1 /$. The correlation between the experimental data and Basquin's law is very good, indicating that Dang Van's and Sines' Criteria may be successfully extended to finite life predictions in the manner described, with a slightly better correlation found in the Dang Van case. To distinguish further between Dang Van's and Sines' criteria, a data set with larger static stress components is needed.

\section{ACKNOWLEDGEMENTS}

The support of EPSRC via a research grant under the Structural Integrity funding initiative is acknowledged. 


\section{REFERENCES}

1. Socie, D. (1987), Multiaxial Fatigue Damage Models. Journal of Engineering Materials and Technology, Transactions of the ASME, Vol. 109, P293-298.

2. Sines, G., Ohgi, G. (1981), Fatigue Criteria Under Combined Stresses or Strains. Jnl. Eng. Mat. and Tech., Trans. ASME, Vol. 103, P82-90.

3. Smith, R.N., Watson, P. and Topper, T.H. (1970), ^ Stress Function for the Fatigue of Metals'. Journal of Materials JMLSA, Vol. 5, P767-778.

4. Fatemi, A. and Socie, D. (1988), A Critical Plane Approach to Multiaxial Fatigue Damage Including Outof-Phase Loading. Fatigue and Fracture of Enginecring Materials and Structures, Vol. 11, P145-165.

5. Dang Van, K., Griveau, B., and Message, O. (1989), On a New Multiaxial Fatigue Limit Criterion: Theory and Applications, Biaxial and Multiaxial fatigue. EGF 3, Edited by M.W. Brown and K.J. Miller, Mechanical Engineering Publications, London, P479-496.

6. Dang Van, K., (1993), Macro-Micro Approach in High-Cycle Multiaxial Fatigue. Advances in Multiaxial Fatigue. ASTM STP 1191. D.L. McDowell and R. Ellis, Eds., American Society for Testing and Materials, Philadelphia, P120-130.

7. Peridas, G., Korsunsky, A., M. and Hills, D., A. (2003), The Relationship between Dang Van Criterion and Traditional Bulk Fatigue Criteria. J. of Strain Analysis for Engineering Design.

8. Peridas, G and Hills, D.A., (2002) Crack Initiation: the Choice of Tests Available to Calibrate Dang Van's Criterion. Fatigue and Fracture of Engineering Materials and Structures\}, Vol. 25, P321-330. 